OPEN ACCESS

Edited by:

Dario Salvi,

Malmö University, Sweden

Reviewed by:

Ivan Miguel Pires,

Universidade da Beira

Interior, Portugal

Palash Chandra Banik,

Bangladesh University of Health

Sciences, Bangladesh

*Correspondence:

Sophia Boudjema sophia.boudjema@hotmail.com

Hervé Tissot Dupont

herve.tissot-dupont@ap-hm.fr

Specialty section:

This article was submitted to

Connected Health

a section of the journal

Frontiers in Digital Health

Received: 04 May 2021

Accepted: 17 September 2021

Published: 21 October 2021

Citation:

Florea O, Gonin J, Tissot Dupont H,

Dufour JC, Brouqui $P$ and

Boudjema S (2021) Internet of Things to Explore Moment 2 of "WHO My

Five Moments" for Hand Hygiene.

Front. Digit. Health 3:684746.

doi: 10.3389/fdgth.2021.684746

\section{Internet of Things to Explore Moment 2 of "WHO My Five Moments" for Hand Hygiene}

\author{
Olga Florea ${ }^{1}$, Jeremy Gonin ${ }^{2}$, Hervé Tissot Dupont ${ }^{1,2 *}$, Jean Charles Dufour ${ }^{3}$, \\ Philippe Brouqui ${ }^{1,2}$ and Sophia Boudjema ${ }^{1 *}$
}

${ }^{1}$ Aix Marseille Université, IRD, MEPHI, IHU-Méditerranée Infection, Marseille, France, ${ }^{2}$ AP-HM, IHU-Méditerranée Infection, Marseille, France, ${ }^{3}$ Aix Marseille Université, AP-HM, INSERM, IRD, SESSTIM, Hop Timone, BioSTIC, Marseille, France

Background: Electronic hand hygiene surveillance systems are developing and considered to be more reliable than direct observation for hand hygiene monitoring. However, none have the capability to assess compliance in complex nursing care.

Materials and Methods: We combined two different technologies, a hand hygiene monitoring system (radiofrequency identification, RFID) and a nursing care recorder at the bedside, and we merge their data to assess hand hygiene performance during nursing. Nursing tasks were classified as standard task procedures or aseptic task procedures corresponding to moment 2 among the five moments for hand hygiene recommended by the WHO. All statistical analyses were performed using R, version 3.6.2. For mixed models, the package "Ime4" was used.

Results: From the merged database over the 2-year study period, 30,164 nursing tasks were identified for analysis, 25,633 were classified as standard task procedures, and 4,531 were classified as aseptic task procedures for nursing care. Hand disinfection with an alcohol-based solution was not detected with our system in $42.5 \%$ of all the recorded tasks, $37 \%$ of all the aseptic task procedures, and $47.1 \%$ of all the standard task procedures for nursing $(p=0.0362)$, indicating that WHO moment 2 was not respected in $37 \%$ of mandatory situations.

Conclusion: Using a combination of different technologies, we were able to assess hand hygiene performance in the riskiest circumstances.

Keywords: hand hygiene (disinfection), IoT - internet of things, five moments for hand hygiene, nosocomial infections, catheter - complications

\section{INTRODUCTION}

It is generally believed that hand hygiene reduces the prevalence of hospital-acquired infections (HAIs), and that inadequate hand hygiene is one of the main risk factors for infection (1-4).

The risk of transmission of microbes from the hands of healthcare workers (HCWs) to patients has been studied extensively in peripheral venous catheters (PVCs), which have long been associated with infection (4-6).

Appropriate hand disinfection prior to PVC insertion significantly reduces the incidence of infection (5-8). Direct observation is the current gold standard to appreciate compliance to hand hygiene. A model of transmission identifying moments at which healthcare workers (HCWs) are at 
risk for transmission has been suggested (9). This model was used to develop "My Five Moments for Hand Hygiene" $(10,11)$. Alcohol-hand-rub (AHR) with an alcohol-based solution before moments 1 and 2 is suggested to prevent the transmission of infection to patients, whereas AHR after moments 3, 4, and 5 is suggested to protect the environment of patients and the transmission of infection to other patients. Moment 2 of the My Five Moments by the WHO is defined as hand disinfection before an aseptic task procedures (12). While Moment 2 appears as a very important clue to cross transmission, its monitoring by direct observation is difficult as events are relatively infrequent compared with moments 1 and 5 .

To bypass this difficulty, we have developed a tool to record the task of a nurse at the bedside of the patient that we called patient smart reader (PSR) (13). This personal digital assistant allows to record nurses care at the bedside and send data in a centralized database connected to hand hygiene monitoring system and available for nurse's feedback in real time.

An automated hand hygiene monitoring system has recently been developed with the aim to replace direct observational monitoring, because it avoids the influence of the Hawthorne effect and appears to be more reliable (14-16). Since 2012, we have developed an automated hand hygiene monitoring system and used it as a part of the HAI Management at the University Hospital Institute Méditerranée Infection (IHU-MI) (17-19). However, to our knowledge, there are no automated hand hygiene monitoring systems that allow for the evaluation of hand hygiene before an aseptic task procedure (moment 2), except for remote video-based surveillance systems, which are time-consuming and not cost-effective $(20,21)$.

This study aimed to evaluate the hand hygiene of nurses before an aseptic task procedure (moment 2) using merge data provided by our automatic hand hygiene monitoring system coupled with the PSR. The hand hygiene monitoring system provided alcoholic hand rub use and entry/exit of the bedroom, and the PSR provided information on the nature of the nursing task recorded during care. This way, we investigated if nurses performed ARH during care and for which kind of care.

\section{MATERIALS AND METHODS}

\section{Electronic Survey}

For this study, we used a combination of data from two different electronic data capture (EDC) systems (see functional schema in Supplementary Material). An EDC system is a computerized system designed for the collection of clinical data in electronic format for use mainly in human clinical trials. The first EDC system was a hand hygiene automated electronic system named MediHandTrace ${ }^{\circledR}$ (MHT), which is an RFID (13.56 MHz)-based personal identifying tracking system that records compliance to AHR for each identified HCW. This system was deployed in a 25-bed infectious disease ward in Marseille, France (18). MHT detects the movements of HCWs by tracking chips that are placed in the shoes of HCWs; RFID signals are emitted by an antenna placed on the floor at the entrance of the bedroom. When an HCW enters the room by opening the door, the device is triggered, and a set of signals are captured and stored in a server at the following time points: [0] when the door opens, [1] when the HCW walks near the antenna, [2] when the HCW walks out of the range of the antenna, and [8] when AHR is performed within or [10] more than $8 \mathrm{~s}$ after the HCW enters the room. The second EDC system was a handy personal digital assistant with a barcode reader named the Patient Smart Reader (PSR), a device that allows to record, at patient bedside, nursing tasks. This tool allows for integration of recorded data within the SQL database stored in the PSR, and are then synchronized with a desktopor server-based database. It allows nursing care tasks and vital signs of patients to be recorded by HCWs in real time during the provision of care (note that an HCW can record the action before or after he/she performs it) (13). This is an important limitation, which explains that only the lack of compliance to AHR can be explored. If AHR is performed, we cannot identify if this is before (good) or after (bad) the task. To record a task, an HCW must identify himself or herself, identify the patient, and identify the task by scanning the barcode affixed to the wall next to the bed. Both systems (MHT and the PSR) are time-synchronized and send the collected data on the same server using a unique ID for each HCW. Together, these two systems enable hand hygiene assessment performed at the time of a specific nursing task.

\section{Selection of Variables}

The nursing tasks that were explored are listed in Table 1. They were classified as standard task procedures (STPs) or aseptic task procedures (ATPs) corresponding to moment 2 for hand hygiene. Each act recorded in a designated aseptic task should be associated with one AHR (hand hygiene opportunity). The lack of compliance to AHR is defined by the ratio of no AHR/number of acts in the designated task.

\section{Data Analyses}

The data used for the study were extracted from the raw data (MHT database and PSR database) collected from January 11, 2017 to January, 11, 2019. The lack of compliance during ATPs was compared with the lack of compliance during STPs. Both systems have been used in the ward for years, and HCWs were invited to participate in the study as part of their natural nursing routine. No specific training was given before the study.

\section{Statistical Analysis}

All the statistical analyses were performed using $\mathrm{R}$, version 3.6.2. For the mixed models, the package "lme4" was used. For all the statistical tests, the alpha risk level was set to $5 \%$, and a bilateral alternative hypothesis (two-sided test) was used, except when the Kolmogorov-Smirnov test was performed with a unilateral alternative hypothesis (one-sided test). The lack of compliance with hand hygiene was compared across durations by the chi-square test.

In a previous study, we reported that the use of an automated electronic surveillance system generated a large amount of data for which the bias in the relationship between HCW activity and HCW performance with AHR was needed to be corrected using a multilevel multivariate logistics model (18). To better control the link between hand hygiene compliance and the type 
TABLE 1 | Ratio of lack of compliance to hand hygiene among 30,164 categorized nursing tasks as surveyed by electronic monitoring system.

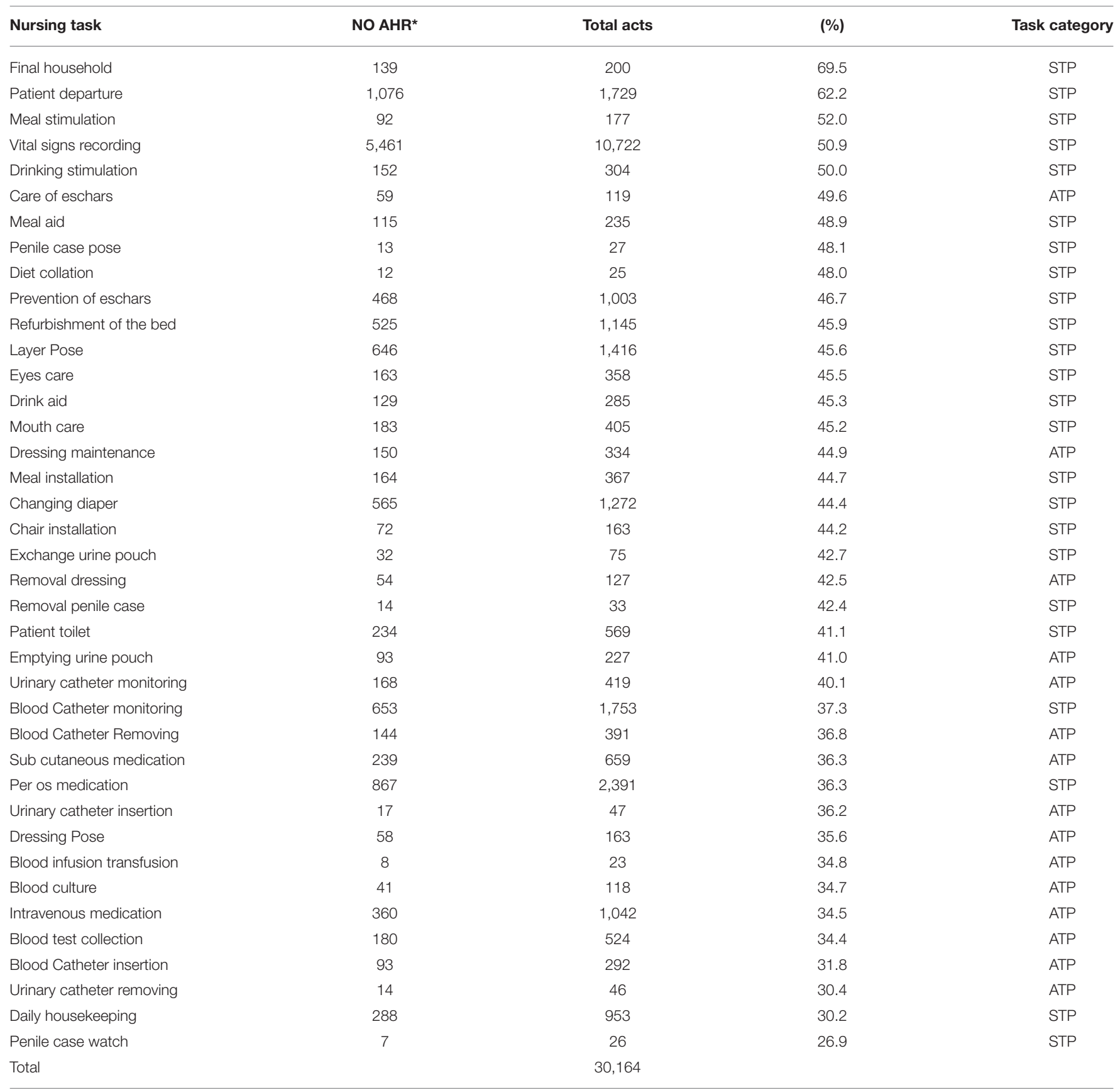

Lack of compliance is defined as no alcoholic hand rub (NO AHR)/ number of dedicated acts recorder with PSR.

*No alcoholic hand rub for task recording.

STP, Standard task procedure; ATP, Aseptic task procedure.

of nursing care, the following generalized linear mixed models were formulated:

$$
\begin{gathered}
M_{0}: \operatorname{logit}\left(P\left(Y_{i j}=1\right)\right)=\beta_{0} \\
M_{0 r}: \operatorname{logit}\left(P\left(Y_{i j}=1\right)\right)=\beta_{0}+b_{0 i} b_{0 i} \sim N\left(0, \sigma_{0}^{2}\right) \\
M_{1}: \operatorname{logit}\left(P\left(Y_{i j}=1\right)\right)=\beta_{0}+\beta_{1} X_{i j}
\end{gathered}
$$

$M_{1 r}: \operatorname{logit}\left(P\left(Y_{i j}=1 \backslash b_{0 i}\right)\right)=\beta_{0}+\beta_{1} X_{i j}+b_{0 i} b_{0 i} \sim N\left(0, \sigma_{0}^{2}\right)$

where $\mathrm{Y}$ is the hand hygiene compliance variable $(\mathrm{Y}=0$ if AHR is not used and $Y=1$ if AHR is used) and $\mathrm{X}$ is the nursing task risk variable ( $\mathrm{X}=0$ if the nursing task is an STP and $\mathrm{X}=1$ if the nursing task is an ATP). The index $\mathrm{i}$ represents the HCW level, $\mathrm{j}$ represents the nursing care level, and $Y_{i j}$ represents hand hygiene compliance. The different models 
were compared using several methods. The Akaike inference criterion (AIC) was used when the models were not nested. For the nested models, comparisons were made with the likelihood ratio test corrected for the comparison between two mixed models. For the models with random effects, the intraclass correlation coefficient (ICC) was used to measure the percentage of variance in AHR use attributable to HCW level. The ICC was estimated based on assumptions for binary variables, and the variance attributable to the HCW level was divided by the total estimate variance. For the models without random effects, the estimations were maximum-likelihood estimations, and for the mixed models (with random effects), the maximumlikelihood estimation method with Laplace approximation was used. Finally, to investigate when AHR was performed within the care sequence, the distribution of hand hygiene within the care sequences was compared between the STPs and ATPs for nursing. The duration of each care sequence was split into 100 intervals of the same length, and the significance was tested by the Kolmogorov-Smirnov test with a unilateral alternative hypothesis (Supplementary Material). The data set is available upon request to the corresponding author.

\section{Ethics}

To ensure the anonymity of the data analyzed, a random number was assigned to the data from each participant included in the database. All the procedures for this study were approved by the Ethics Committee of our institution ( $\mathrm{N}^{\circ}$ 2016-018). Before the study, the HCWs were informed of the details of the study and they gave their consent to be monitored by the automated systems.

\section{RESULTS}

Among 39 nursing procedures, 24 were classified as standard task procedures (STPs) and 15 were classified as aseptic task procedures (ATPs) for which ARH before the procedure is mandatory (Moment 2). The most frequent procedures, such as recording of vital signs, per os medication, and layer pose were STPs. Among the ATPs, blood catheter monitoring, intravenous medication, and blood test collection were the most frequent. Our system identified the HCW in the patient room for 30,164 nursing tasks procedures during the 2 -year study period. Of the 30,164 tasks, 25,633 were classified as requiring STPs for nursing, and 4,531 were classified as requiring ATPs for nursing (Table 1).

No AHR was detected in $42.5 \%$ of all the tasks, $37 \%$ of the ATPs, and $47.1 \%$ of the STPs for nursing $(p=0.0362)$, indicating that moment 2 was not respected in $37 \%$ of the mandatory situations. For the ATPs, the nurses performed better than the assistant nurses (35.9 vs. $51.6 \%$ of lack of compliance to M2; $p<0.001)$. The assistant nurses practicing STPs did not perform AHR at all in $52 \%$ of these nursing tasks. The housekeeping workers only performed STPs, and AHR was not detected in $40.6 \%$ of the tasks. Among the nursing tasks performed, those with lower lack of AHR execution was urinary catheter removal, blood catheter insertion, blood sample collection, intravenous medication, and blood cultures (Figure 1). Interestingly, some tasks that were considered STPs for nursing, such as penile case assessments and per-os medication, were associated with higher AHR performance. Figure 2 shows the distribution of AHR events among 16,416 nursing tasks, 12,759 classified STPs (in gray), and 2,853 ATPs (in black) during the nursing task. Hand hygiene is more frequently performed at the end of the task when STPs nursing is performed (Kolmokorov-Smirnov $0.84 p<2.2 \mathrm{E}-16)$. In ATPs nursing the AHR is performed in a similar way upon entry rather than upon exit. A multilevel analysis showed that the variables associated with AHR were the behavior of HWCs itself and the nature of the tasks. AHR was slightly performed more often by any of the HCWs when the task recorded was an ATP [ORa 1.08 (95\% CI) $(1.01-1.17) p=0.036]$ (Supplementary Material).

\section{DISCUSSION}

Electronic hand hygiene systems have been developed not only to record but also to promote compliance. These systems have been designed to ensure that HCWs perform hand hygiene before approaching the patient's bedside (M1) and to issue an alert for HCWs to do so (18). Despite their advantages, newer technologies, at this time, are unable to differentiate the five moments for hand hygiene, but most of them can detect whether the hands of HCWs have been disinfected before touching a patient (moment 1 ) and before leaving the patient zone (similar to moments 3, 4, and 5). Nevertheless, the level of risk of infection is associated with specific steps in the care process and the relative importance of hand hygiene at each of the five moments in preventing microbial transmission and infection outcomes is still unknown (22). According to the WHO guidelines, it is assumed that "an ideal indicator of hand hygiene performance would reliably capture each moment requiring hand hygiene, even during complex care activities" (20). In this study, by merging the data obtained from the two systems, we successfully identified that in ATPs that are identified as high risk for cross-transmission of microbes to patients, no ARH was performed in $37 \%$ of the tasks. However, this lack of compliance may be underestimated. Among task for which we detect AHR it is possible that hand hygiene was performed after the aseptic procedure as reported in Figure 2, and consequently considered as inefficient. This is the main limitations of our study.

The lack of AHR among the nurses practicing ATPs is likely related to the fact that they have reduced the need for hand hygiene by wearing non-sterile gloves, which can make HCWs feel less exposed to dirt and microbes, and protected against blood-borne diseases (20). The overuse of gloves has been shown to be one major factor explaining poor hand hygiene compliance $(23,24)$. There is currently increasing scientific evidence that glove disinfection is as effective in preventing infections in experiments as in routine care, and that glove disinfection should be promoted $(25,26)$. Recently, Fehling et al. reported that allowing glove disinfection significantly improved hand disinfection, particularly at moment 2 , and reduced the occurrence of severe infections considerably (27). The weakness of our system is that we detect the lack of 


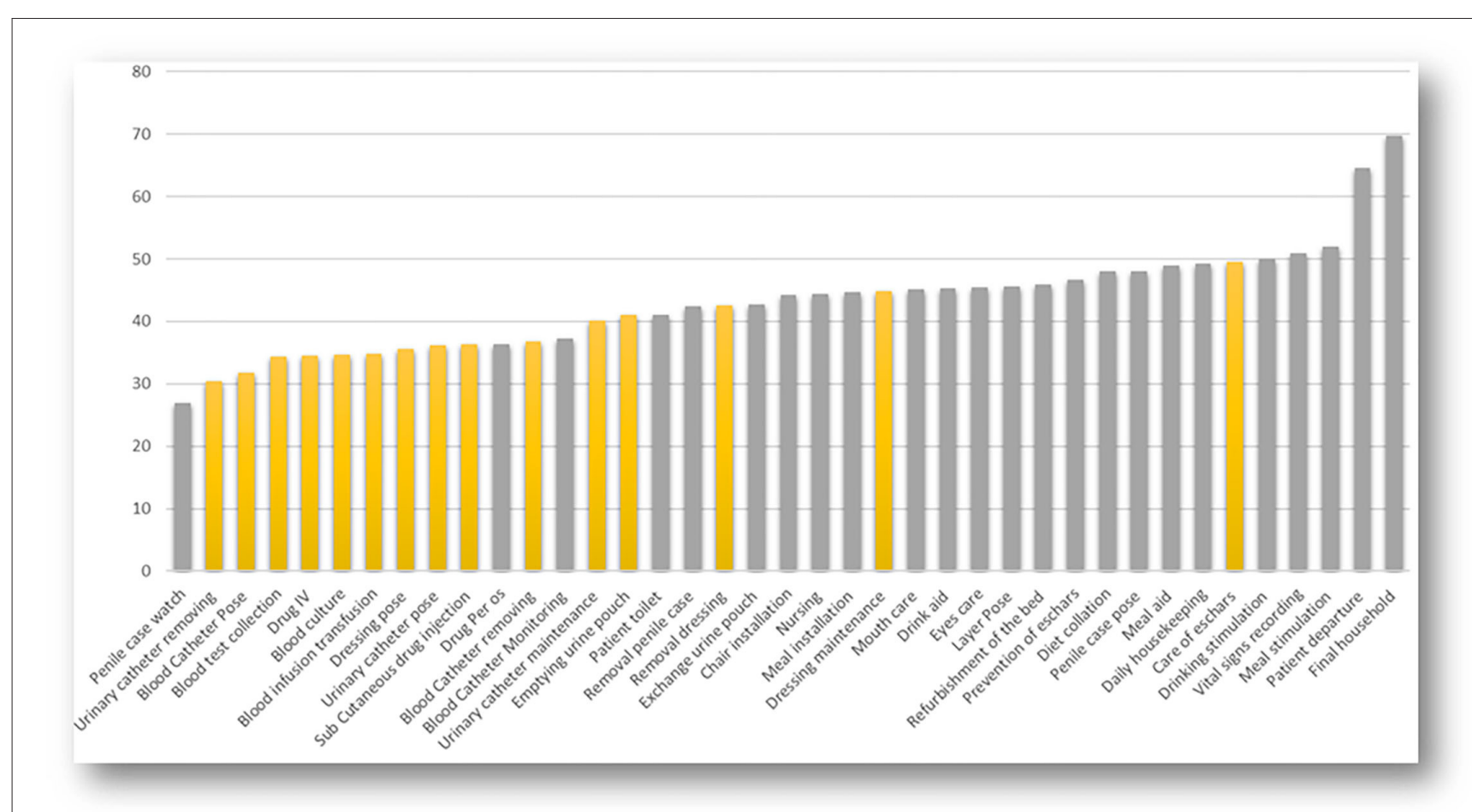

FIGURE 1 | Distribution of lack of compliance (\%) to hand hygiene in aseptic task procedure (WHO Moment 2) vs. standard task procedure nursing. Hand hygiene is better performed when the nursing is in the category aseptic task (yellow) compare to standard precaution (gray) that were 1,678 (37\%)/12,070 (47.1\%) $p<2.2 \mathrm{E}-16$.

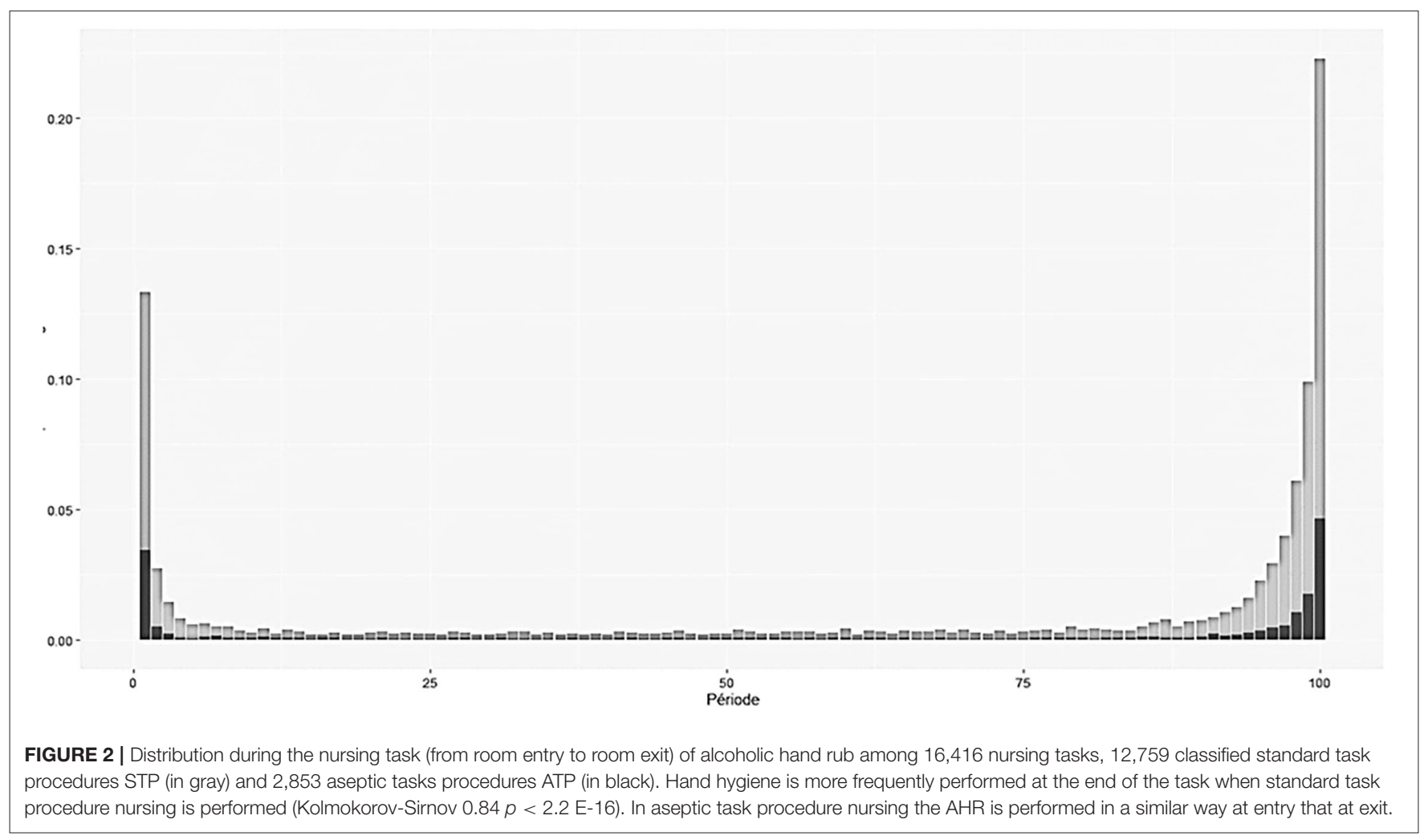


AHR, but the technology available at this time is unable to detect AHR and whether it occurs precisely within the time of nursing. Only video capture is capable of doing it. A new generation of hand hygiene monitoring should evolve to dematerialized video system with real time data analysis and feedback.

In conclusion, assessing hand hygiene surveillance during complex care is feasible by combining different technologies. Although not perfectly responding to WHO requirements for an ideal indicator, this proof-of-concept study reveals that AHR is not performed in at least $37 \%$ of care situation for which it is mandatory. Focused interventions on the practice of moment 2 for hand hygiene should be quicky implemented and then evaluated.

\section{DATA AVAILABILITY STATEMENT}

The original contributions presented in the study are included in the article/Supplementary Material, further inquiries can be directed to the corresponding author/s.

\section{ETHICS STATEMENT}

This study has been approved by the Ethical Review Board of IHU Mediterranée-Infection under $N^{\circ}$ 2016-018.

\section{REFERENCES}

1. Mathur P. Hand hygiene: back to the basics of infection control. Indian J Med Res. (2011) 134:611-20. doi: 10.4103/0971-5916.90985

2. Peters A, Palao VC, Lotfinejad N, Pittet D. WHO year of the nurse and midwife: more clean and educated hands for all. J Infect Prev. (2020) 21:166-9. doi: 10.1177/1757177420958042

3. Loveday HP, Wilson JA, Pratt RJ, Golsorkhi M, Tingle A, Bak A, et al. epic3: national evidence-based guidelines for preventing healthcare-associated infections in NHS hospitals in England. J Hosp Infect. (2014) 86 Suppl 1:S1-70. doi: 10.1016/S0195-6701(13)60012-2

4. Keleekai NL, Schuster CA, Murray CL, King MA, Stahl BR, Labrozzi LJ, et al. Improving nurses' peripheral intravenous catheter insertion knowledge, confidence, and skills using a simulation-based blended learning program: a randomized trial. Simul Healthc. (2016) 11:376-84. doi: 10.1097/SIH.0000000000000186

5. Zhang L, Cao S, Marsh N, Ray-Barruel G, Flynn J, Larsen E, et al. Infection risks associated with peripheral vascular catheters. J Infect Prev. (2016) 17:207-13. doi: 10.1177/1757177416655472

6. Gould CV, Umscheid CA, Agarwal RK, Kuntz G, Pegues DA, Healthcare Infection Control Practices Advisory Committee. Guideline for prevention of catheter-associated urinary tract infections 2009. Infect Control Hosp Epidemiol. (2010) 31:319-26. doi: 10.1086/651091

7. Kampf G, Reise G, James C, Gittelbauer K, Gosch J, Alpers B. Improving patient safety during insertion of peripheral venous catheters: an observational intervention study. GMS Hyg Infect Control. (2013) 8:Doc18. doi: 10.3205/dgkh000218

8. McCalla S, Reilly M, Thomas R, McSpedon-Rai D, McMahon LA, Palumbo M. An automated hand hygiene compliance system is associated with decreased rates of health care-associated infections. Am J Infect Control. (2018) 46:1381-6. doi: 10.1016/j.ajic.2018.05.017

9. Pittet D, Allegranzi B, Sax H, Dharan S, Pessoa-Silva CL, Donaldson L, et al. Evidence-based model for hand transmission during patient

\section{AUTHOR CONTRIBUTIONS}

$\mathrm{PB}$, JD, and SB conceived and designed the experiments. OF performed the experiments. OF, JG, and SB analyzed the data. OF, JG, and PB wrote the manuscript. OF, JD, and SB edited the manuscript. All authors contributed to the article and approved the submitted version.

\section{FUNDING}

This study was funded by ANR-15-CE36-0004-01 and by ANR Investissements d'avenir, Mediterranée infection 10-IAHU-03.

\section{ACKNOWLEDGMENTS}

We would like to thank the nurses, assistant nurses, and housekeeping personnel from the University Hospital Institute Méditerranée-Infection who kindly participated in the study. English was edited by an English native, Maria Filannino. The plagiarism rate was assessed by Magister by Compilatio.net to be $3 \%$.

\section{SUPPLEMENTARY MATERIAL}

The Supplementary Material for this article can be found online at: https://www.frontiersin.org/articles/10.3389/fdgth. 2021.684746/full\#supplementary-material

care and the role of improved practices. Lancet Infect Dis. (2006) 6:641-52. doi: 10.1016/S1473-3099(06)70600-4

10. Sax H, Allegranzi B, Uçkay I, Larson E, Boyce J, Pittet D. 'My five moments for hand hygiene': a user-centred design approach to understand, train, monitor and report hand hygiene. J Hosp Infect. (2007) 67:9-21. doi: 10.1016/j.jhin.2007.06.004

11. Salmon S, Pittet D, Sax H, McLaws ML. The "My five moments for hand hygiene" concept for the overcrowded setting in resource-limited healthcare systems. J Hosp Infect. (2015) 91:95-9. doi: 10.1016/j.jhin.2015.04.011

12. Pittet D, Allegranzi B, Boyce J. Experts WHOWA for PSFGPSCCG of. The World Health Organization Guidelines on Hand Hygiene in Health Care and Their Consensus Recommendations. Infect Control Hosp Epidemiol. (2009) 30:611-22. doi: 10.1086/600379

13. Florea O, Dufour J-C, Magnin C, Brouqui P, Boudjema S. Improving health care workers compliance with traceability by recording the nursing process at the point of care using a personal digital assistant with a barcode. J Nurs Care. (2020) 9:500. doi: 10.1101/2020.01.14.20017434

14. McCambridge J, Witton J, Elbourne DR. Systematic review of the Hawthorne effect: new concepts are needed to study research participation effects. J Clin Epidemiol. (2014) 67:267-77. doi: 10.1016/j.jclinepi.2013.08.015

15. Hagel S, Reischke J, Kesselmeier M, Winning J, Gastmeier P, Brunkhorst FM, et al. Quantifying the hawthorne effect in hand hygiene compliance through comparing direct observation with automated hand hygiene monitoring. Infect Control Hosp Epidemiol. (2015) 36:957-62. doi: 10.1017/ice. 2015.93

16. Bruchez SA, Duarte GC, Sadowski RA, Custódio da Silva Filho A, Fahning WE, Belini Nishiyama SA, et al. Assessing the Hawthorne effect on hand hygiene compliance in an intensive care unit. Infect Prev Pract. (2020) 2:100049. doi: 10.1016/j.infpip.2020.100049

17. Brouqui P, Boudjema S, Soto Aladro A, Chabrière E, Florea O, Nguyen H, et al. New approaches to prevent healthcare-associated infection. Clin Infect Dis Off Publ Infect Dis Soc Am. (2017) 65(Suppl.1):S50-4. doi: 10.1093/cid/ cix433 
18. Dufour J-C, Reynier P, Boudjema S, Soto Aladro A, Giorgi R, Brouqui P. Evaluation of hand hygiene compliance and associated factors with a radio-frequency-identification-based real-time continuous automated monitoring system. J Hosp Infect. (2017) 95:344-51. doi: 10.1016/j.jhin.2017. 02.002

19. Boudjema S, Dufour JC, Aladro AS, Desquerres I, Brouqui P. MediHandTrace ${ }^{\circledR}$ : a tool for measuring and understanding hand hygiene adherence. Clin Microbiol Infect. (2014) 20:22-8. doi: 10.1111/1469-0691. 12471

20. Boudjema S, Reynier P. Hand hygiene analyzed by video recording. J Nurs Care. (2016) 5:338. doi: 10.4172/2167-1168.1000338

21. Boudjema S, Tarantini C, Peretti-Watel P, Brouqui P. Merging video coaching and an anthropologic approach to understand health care provider behavior toward hand hygiene protocols. Am J Infect Control. (2017) 45:487-91. doi: 10.1016/j.ajic.2016.12.016

22. Boyce JM. Electronic monitoring in combination with direct observation as a means to significantly improve hand hygiene compliance. Am J Infect Control. (2017) 45:528-35. doi: 10.1016/j.ajic.2016.11.029

23. Fuller C, Savage J, Besser S, Hayward A, Cookson B, Cooper B, et al. "The dirty hand in the latex glove": a study of hand hygiene compliance when gloves are worn. Infect Control Hosp Epidemiol. (2011) 32:1194-9. doi: 10.1086/ 662619

24. Vikke HS, Vittinghus S, Giebner M, Kolmos HJ, Smith K, Castrén M, et al. Compliance with hand hygiene in emergency medical services: an international observational study. Emerg Med J. (2019) 36:171-5. doi: 10.1136/ emermed-2018-207872

25. Kampf G, Lemmen S. Disinfection of gloved hands for multiple activities with indicated glove use on the same patient. J Hosp Infect. (2017) 97:3-10. doi: 10.1016/j.jhin.2017.06.021
26. Vogel A, Boudjema S, Brouqui P. Disinfection of gloved hand during routine care. New Microb New Infect. (2021) 41:100855. doi: 10.1016/j.nmni.2021. 100855

27. Fehling P, Hasenkamp J, Unkel S, Thalmann I, Hornig S, Trümper L, et al Effect of gloved hand disinfection on hand hygiene before infection-prone procedures on a stem cell ward. J Hosp Infect. (2019) 103:321-7. doi: 10.1016/ j.jhin.2019.06.004

Conflict of Interest: PB owned part of the start-up company MedihandTrace SAS, which commercialized the electronic monitoring system.

The remaining authors declare that the research was conducted in the absence of any commercial or financial relationships that could be construed as a potential conflict of interest.

Publisher's Note: All claims expressed in this article are solely those of the authors and do not necessarily represent those of their affiliated organizations, or those of the publisher, the editors and the reviewers. Any product that may be evaluated in this article, or claim that may be made by its manufacturer, is not guaranteed or endorsed by the publisher.

Copyright (C) 2021 Florea, Gonin, Tissot Dupont, Dufour, Brouqui and Boudjema. This is an open-access article distributed under the terms of the Creative Commons Attribution License (CC BY). The use, distribution or reproduction in other forums is permitted, provided the original author(s) and the copyright owner(s) are credited and that the original publication in this journal is cited, in accordance with accepted academic practice. No use, distribution or reproduction is permitted which does not comply with these terms. 